\title{
MEASUREMENT OF LENS POWERS AND DESIGN OF TRIAL CASE LENSES
}

NEARLY 50 years have elapsed since the design of trial case lenses was last considered by an authoritative body in Great Britain. Since that time several factors have emerged to complicate what was then a simple matter. First, a new method of measuring lens powers, embodied in the vertex focimeter, has been adopted by many practitioners. This instrument gives results which may differ to an appreciable extent from those obtained by the traditional process of neutralization. For example, using different methods, it would be quite possible for two practitioners to disagree by over one dioptre in their measurement of a strong positive lens. Secondly, the use of curved lenses has become increasingly widespread, and this means that the lenses dispensed will usually differ in form from the trial lenses used in testing. Thirdly, the introduction of various plano-form trial cases with disparate systems of numbering has created a further source of possible discrepancy between the focal effect intended by the prescriber and that of the lenses actually dispensed.

The time has clearly been ripe for a review of the whole problem. It was for this reason that the then Minister of Health, early in 1955, appointed a Committee under the chairmanship of Sir Stewart Duke-Elder "to review standards for trial case lenses and the method of measuring lens powers and to make recommendations". The members of the Committee included representatives of all the professional bodies and trade associations concerned, and their Report, which was unanimous, has recently been published (Ministry of Health, 1956).

The following is a brief summary of the contents of the report. Although the subject matter may appear to be uncomfortably involved in optical mathematics, it is nevertheless of direct concern to every ophthalmic practitioner as well as to dispensing opticians and lens manufacturers.

Measurement of Lens Powers.-Part I of the Report deals with the measurement of lens powers. It is obviously desirable that the method adopted should assign the same numerical value to lenses having the same focal effect (at the same distance from the eye) even though they differ substantially in form, thickness, or both. This requirement is satisfied by making the criterion the state of convergence or divergence of the rays of light as they emerge from the back surface of the lens: in other words, by numbering lenses according to their back vertex powers. Another essential requirement is that trial case lenses should be numbered on the same basis 
as lenses supplied to prescription. Nearly all the trial cases made in Great Britain during the past 40 years have been numbered on systems which are either identical with or closely approximate to a back vertex numbering. Hence, a back vertex numbering for all ophthalmic lenses is not only best $\stackrel{\vec{s}}{\vec{s}}$ in theory but is needed to ensure conformity with the great majority of trial cases now in use.

Despite its many conveniences, neutralization does not afford an accurate $\frac{\widehat{T}}{\vec{\phi}}$ means of measuring the back vertex power of curved lenses. To obtain central contact the neutralizing lens is necessarily placed against the convex surface of the lens under test. The quantity so measured is the front vertex power. $\stackrel{\circ}{\rightarrow}$ Unfortunately, the front vertex power of a strong positive lens may differ $\vec{\omega}$ considerably from its back vertex power. Furthermore, two lenses having the same front vertex power may have different back vertex powers. Thus, neutralization is open to two objections: first, it does not measure the quantity of real significance, and, secondly, its results are not readily con- $\stackrel{\vec{D}}{\mathrm{o}}$ vertible into back vertex powers. The relationship is a complicated one, $\vec{\omega}$ involving both the form and the thickness of the lens. Table I of the Report 9 shows the difference between the two powers for a representative range of $\vec{P}$ lenses, indicating that neutralization may lead to appreciable errors when applied to convex lenses over $+2 \mathrm{D}$.

In recommending that the back vertex system of measuring lens powers now become an accepted standard, the Committee points out that it hog already been adopted in most other countries and by industry generally Great Britain. At least four British firms have produced vertex focimete suitable for professional use.

Importance of the Vertex Distance.-The effective power of a spectacle $\stackrel{\circ}{\circ}$ lens at the wearer's eye is influenced by the "vertex distance", that is to say, $\overrightarrow{0}$ the distance from the back vertex of the lens to the cornea. To a first 3 approximation, each millimetre of variation in the vertex distance alters the effective power of a lens by $F^{2} / 1,000$, where $F$ denotes its nominal power. Thus, a 3-mm. variation in the vertex distance would alter the effective power of a $16 \mathrm{D}$ lens by approximately $0.75 \mathrm{D}$.

The design of trial frames does not always permit the back lens to be placed as close to the eye as the lens which will subsequently be worn. In $₹$ this event there will be a change in the vertex distance. Table II of the 9 Report shows the effect of such changes for lenses of various powers, from $\frac{D}{0}$ which the Committee concludes that a prescription of high power is not complete unless the vertex distance is stated. The Committee accordingly $N$ recommends that the vertex distance be recorded whenever the prescribed $\tilde{O}$ power exceeds $5 \mathrm{D}$ and even on lower powers when a significant change in $\underset{\omega}{\mathcal{\omega}}$ the vertex distance may be expected. It is considered to be the duty of the ${ }^{\circ}$ dispenser to determine any such change and to modify the prescribed power accordingly. 
Standards for Trial Case Lenses.-Part II of the Report deals with trial case lenses, beginning with a brief review of two previous standards.

The first was that published in 1908 by the Optical Society of London. It was then recommended that the spherical lenses should be made equiconvex and concave, the system of numbering being based on neutralization. The negative lenses were to be marked with their equivalent power, which differs but little from the vertex power when the centre thickness of the lens is small. The positives were to neutralize the corresponding negatives. As shown by Table III of the Report, this system approximates very closely to a back vertex numbering. Most of the full-aperture trial cases since made in Great Britain have followed the recommendations of the 1908 standard.

In 1927, new standards for trial case lenses were published jointly by the Institute of Ophthalmic Opticians and the Associations of Wholesale and Manufacturing Opticians. The sponsors recommended that the spherical lenses be made plano-convex and concave and of $25 \mathrm{~mm}$. effective aperture. It was further recommended that the positives should continue to neutralize the corresponding negatives, as the Optical Society had proposed in 1908. The authors of the new standard evidently failed to realize that, although the same numbering system was apparently being continued, its practical effect would be completely upset by the change of lens form.

For example, a $+20 \mathrm{D}$ lens made to the Optical Society's standard has a back vertex power of very nearly $+19.87 \mathrm{D}$. Made to the new standard, it would have a back vertex power of $+21.25 \mathrm{D}$ if the plane surface were placed next to the eye as was obviously intended. Only by placing the positive lenses with the curved surface next to the eye could their back vertex powers be made to coincide with the marked powers.

This fact has been appreciated by several manufacturers, whose planoform trial lenses are so engraved that the curved surface would invariably be placed next to the eye. Other manufacturers, however, engrave the opposite side of the rim with the intention that the plane surface of the positives should be placed towards the eye. As shown by Table IV of the Report, the discrepancy between the two systems begins to assume significance when the marked power exceeds $+10 \mathrm{D}$.

Form of Trial Lenses.-The Report just published deals exhaustively with the difficult problem of deciding on the ideal form for trial lenses.

Whatever the basis on which the individual lenses are numbered, it is their form and thickness, together with the separation between them and the order in which they are placed in the trial frame, that determines the effective. power of a combination of lenses. In general, the back vertex power of a trial lens combination is not the simple sum of the marked powers, and in some cases the discrepancy may be significant. A further complication is that two lenses or lens combinations having the same back vertex power (and, therefore, equally effective for distant vision) may not be equally 
effective for near vision. This, again, is a matter involving the form and thickness of the lenses.

The Report presents, in graphical form, the results of an extensive analysis of the effective powers of various trial lens combinations of different designs, both for distant and near vision. In the light of these results the Committee feels unable to recommend, as an exclusive standard, any of the existing series of trial case lenses because none is clearly superior to all others in every respect.

The Committee took note of two different principles of design which have been put forward on earlier occasions.

One is the principle of "direct substitution" advocated by Swaine (1923). The design favoured by this author was based on the proposition that the single correcting lens substituted for a trial lens combination would most nearly reproduce the same effect if, in addition to a common back vertex numbering, there was also a similarity of form and thickness.

The other principle, that of "additive vertex powers", is embodied in $\frac{\vec{\sigma}}{\omega}$ certain trial cases manufactured in the United States. This basis of design 9 has the advantage that the back vertex power of any sphero-cylindrical combination is given quite accurately by the marked powers of the lenses. Unfortunately, this design is not equally satisfactory from the standpoint of effective powers in near vision.

The Committee points out the possibility of reconciling these hithert $\delta$ conflicting principles of design by inverting the usual order in which the lenses are placed in the trial-frame. It is recognized that if the cylinder is placed behind the sphere, some means of reading the axis would have to be provided. In the expectation that this mechanical difficulty could easily be overcome, the Committee recommends manufacturers to consider the possibilities of a trial case designed as described in the Report.

Numbering of Trial Lenses.-The Committee recommends that a back vertex numbering should be applied to all trial case lenses. Where the form of the lens is such that the back vertex power differs from the front vertex or neutralizing power, the latter should also be stated.

The Committee points out that the back vertex power of any combination of lenses in a trial frame can be readily ascertained by means of a vertex focimeter. Discrepancies of effective power arising from whatever source, including a front vertex numbering where this applies to certain existing trial sets, can thereby be eliminated.

Prism Units.-The Committee, while noting one theoretical advantage of $N$ the centrad as the unit of prism power, considers that the prism dioptre is $N$ so convenient and well-established that it should now be adopted exclusively. It further recommends that the use of older units such as degrees of apical 0 angle and degrees of deviation should be discontinued.

One complication which arises with the stronger plano prisms in a trial 
case is that the deviation is appreciably affected by the angle of incidence. In the 1908 standard it was recommended that the minimum deviation be taken as the basis of numbering. The Committee considers, however, that, for reasons explained, the deviation should be measured when the light is incident normally at one surface. Table $\mathrm{V}$ of the Report, compiled for the convenience of manufacturers, gives details of the apical angles required (for glass of the standard refractive index of 1.523) to comply with the basis of numbering recommended. It shows, for example, that a prism marked $15 \triangle$ (the deviation for light incident or emergent normally) would need an apical angle of $15^{\circ} 32^{\prime}$ and would have a minimum deviation of $14 \cdot 44 \triangle$.

Marking of Lenses and Tolerances.-The Report proceeds to formulate standards of quality and accuracy, detailing the limits of permissible errors in lens and prism powers, axis and base-apex directions, and optical centration. In framing these recommendations the Committee took note of parallel discussions which were then taking place through the British Standards Institution and which have since resulted in the publication of British Standard No. 2738: "Spectacle Lenses" (1956), as discussed by Cridland (1956).

It was felt that, in general, tolerances for trial case lenses and prisms might justifiably be narrower than those which had been deemed appropriate for lenses supplied to prescription. On the other hand, the Committee points out that unreasonably close tolerances would merely add to the cost of a trial case without bringing any real benefits in return.

Laboratory Testing of Lenses.-It is interesting to recall that the National Physical Laboratory actively assisted in the preparation of the 1908 standard and has rendered a service to industry ever since in calibrating trial lenses submitted for test. At the request of the Laboratory's representative, the Committee considered whether standard conditions should be laid down for future tests.

It was agreed that light of a specified wavelength must be used because the power of a lens made from hard crown glass varies by approximately 2 per cent. over the range of the visible spectrum. The Committee recommends, in line with current industrial practice, that the wavelength chosen should be 5,875.6 Angstrom units, corresponding to the ' $d$ ' line of the helium spectrum.

A further point considered was whether a standard aperture should be fixed for purposes of testing. If the aperture is unduly restricted, the depth of focus is increased and the experimental determination of the focal length becomes correspondingly uncertain. On the other hand, if the aperture is increased, the effects of spherical aberration may become obtrusive. After consideration, the Committee came to the conclusion that it would be unwise to specify one standard aperture for laboratory testing, and that the 
National Physical Laboratory should in each case select the aperture calculated to result in the most accurate determination of the paraxial power of the lens.

In the concluding sections of the Report, the Committee draws attention $\overrightarrow{\vec{s}}$ to two factors affecting the accuracy of vertex focimeter readings.

\section{REFERENCES}

British Standard (1956). No. 2738 "Spectacle Lenses".

CRIDLAND, N. (1956). British Journal of Ophthalmology, 40, 611.

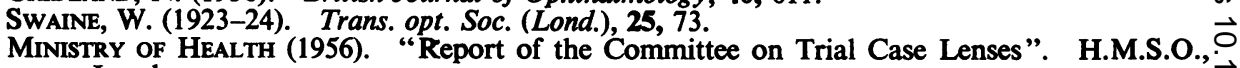
London.

London.

